\title{
Towards future pedestrian-vehicle interactions: Introducing theoretically-supported AR prototypes
}

\author{
Wilbert Tabone \\ Delft University of Technology, \\ Netherlands \\ w.tabone@tudelft.nl
}

\author{
Riender Happee \\ Delft University of Technology, \\ Netherlands \\ r.happee@tudelft.nl
}

\author{
Natasha Merat \\ University of Leeds, United Kingdom \\ n.merat@its.leeds.ac.uk
}

\begin{abstract}
The future urban environment may consist of mixed traffic in which pedestrians interact with automated vehicles (AVs). However, it is still unclear how AVs should communicate their intentions to pedestrians. Augmented reality (AR) technology could transform the future of interactions between pedestrians and AVs by offering targeted and individualized communication. This paper presents nine prototypes of AR concepts for pedestrian-AV interaction that are implemented and demonstrated in a real crossing environment. Each concept was based on expert perspectives and designed using theoretically-informed brainstorming sessions. Prototypes were implemented in Unity MARS and subsequently tested on an unmarked road using a standalone iPad Pro with LiDAR functionality. Despite the limitations of the technology, this paper offers an indication of how future AR systems may support future pedestrian-AV interactions.
\end{abstract}

\section{CCS CONCEPTS}

- Human-centered computing; • Human computer interaction (HCI); • Interaction paradigms; • Mixed / augmented reality;

\section{KEYWORDS}

augmented reality, pedestrian-automated vehicle interaction, implemented prototypes, road crossing, theoretically-informed design

ACM Reference Format:

Wilbert Tabone, Yee Mun Lee, Natasha Merat, Riender Happee, and Joost de Winter. 2021. Towards future pedestrian-vehicle interactions: Introducing theoretically-supported AR prototypes. In 13th International Conference on Automotive User Interfaces and Interactive Vehicular Applications (AutomotiveUI '21), September 09-14, 2021, Leeds, United Kingdom. ACM, New York, NY, USA, 10 pages. https://doi.org/10.1145/3409118.3475149

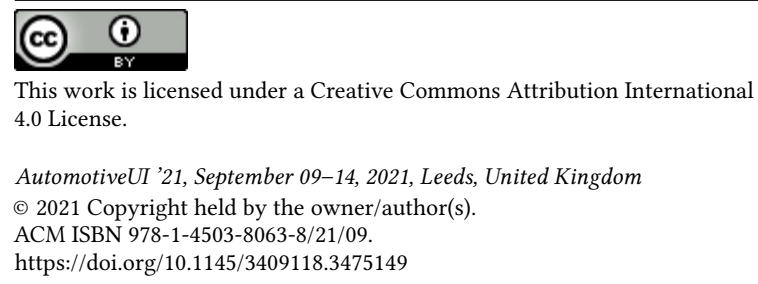

\author{
Joost de Winter \\ Delft University of Technology, \\ Netherlands \\ j.c.f.dewinter@tudelft.nl
}

\section{INTRODUCTION}

Future automated vehicles (AVs) have to be able to drive in complex environments containing many interaction partners, including vulnerable road users (VRUs). In recent years, the 'control loop', in which the automated vehicle locates itself, perceives its surroundings, and decides upon the best trajectory, has changed into an 'interaction loop', where multiple road users cooperate through the wireless exchange of information [57]. To close the interaction loop, the AV may need to communicate its intentions to VRUs, who traditionally received such information explicitly from the driver and implicitly through vehicle kinematics [31]. Current solutions are smart infrastructure and vehicle-mounted external human-machine interfaces (eHMIs) [7, 10, 36, 51], and see [8] for a review of $70 \mathrm{eHMI}$ concepts. However, these interfaces may be hard to read from a distance and potentially ambiguous, especially when encountering multiple pedestrians, as it could be unclear to whom the AV is communicating [46].

AR has already been used to support operators in many domains, including the military [32, 55], museums [52], industry [35], and entertainment [24]. As AR technology becomes more context-aware and pervasive [20], AR may benefit pedestrians. Through AR, it becomes possible to remove the display from the vehicle and place it anywhere in the world. Since each pedestrian would receive individualised communication, AR could solve the one-to-many communication problem of eHMIs [53]. Additionally, AR may prove advantageous in terms of costs, as it may be cheaper to build virtual interfaces than it would be to build their physical counterparts such as eHMIs.

AR has already been employed in traffic research, for example, to provide drivers with navigation advice in the form of augmented road signs [49] or to inform the driver about safe and unsafe slots via green and red coloured road surfaces [63]. Previous research on AR for VRUs has focused on handheld devices $[28,54]$ and AR glasses [40] for pedestrian route guidance and navigation. Furthermore, a 'pedestrian in the loop' system that uses the HoloLens to augment vehicles in the real environment for testing safety-critical situations has been developed by Hartmann et al. [22], whereas Kamalasanan et al. [25] conducted a HoloLens experiment to investigate the effect of an AR traffic light on pedestrian behaviour. Additionally, AR concepts that aim to provide crossing advice to VRUs have been presented as superimposed layers on a photo of the streetscape [23] and as part of a VR simulation [45]. However, to date, the literature 
presents only few AR prototypes for pedestrian-AV interactions in a real environment. Furthermore, the literature offers little guidance regarding the question: how should such prototypes function and look? This paper attempts to address this question by presenting $\mathrm{AR}$ prototypes for pedestrian-AV interactions that function in a real environment. The prototypes were designed using a theoretically informed approach that draws upon fundamental principles and concepts such as affordances [17], the field of safe travel [19], and risk perception [50]. In the spirit of open science, the code behind the AR concepts is provided as supplementary material (https://doi. org/10.4121/14933082) to facilitate reproducibility and encourage further development.

In this paper, we present nine AR concepts that aim to support pedestrians that want to cross the road. The concepts were derived by building upon interviews with human factors experts [53] and supported by theories of perception, human factors, and spatial computing.

\section{METHODS}

A four-phase process was employed to create the AR prototypes. In Phase 1, expert perspectives were analysed to extract AR design ideas. Phase 2 entailed identifying literature that served as theoretical background and inspiration for AR design. The next phase included an iterative design [43] brainstorming process that involved discussions on design considerations and sketching, followed by a heuristic evaluation. Phase 4 covered the implementation of the sketched concepts.

\subsection{Phase 1: Collection of ideas}

In Tabone et al. [53], experts in human factors provided their views on automated driving in future urban environments, eHMIs, and the use of AR in future traffic. For the present study, the experts' statements were extracted from [53] and its corresponding interview transcripts to identify concepts for pedestrian-AV interaction using AR. The analysis yielded seven quotes from the experts that could be turned into implementable concepts:

1. "It would be ideal to provide a unified affordance of the intentions of the vehicle, rather than just a visual colouring. For example, it would be interesting to alter the perceived surface of the AV to make it appear as more or less threatening, contingent on need." (P. A. Hancock, [53, p. 6]).

2. "Make objects stand out using subjective contours." (Peter A. Hancock, personal correspondence with Joost C. F. de Winter based on Hancock [21]).

3. "The AR system should provide information related to safety. The pedestrian could be presented with safety corridors related to which vehicles will stop for them. The advantage of this safety corridor concept is that it is integrating information from several vehicles. In doing so, it is clear to whom the vehicles are communicating, in contrast to the undirected communication of traditional eHMIs." (Martin Baumann, [53, p. 4]). "AR glasses may allow for hands-free navigation and assist pedestrians with speed estimations by projecting the AV's trajectory." (Neville A. Stanton, [53, p. 11]).
4. "AR should not overwhelm the user. Information should include highlighting of hazards such as specific alerts that a vehicle is approaching." (Shuchisnigdha Deb, [53, p. 5]).

5. "Augmented 3D traffic light in the form of a virtual fence to stop pedestrians from crossing a vehicle lane. It would be ideal for tram lanes as well, and simple enough for a child to understand even in ambiguous situations when multiple pedestrians are present." (Riender Happee, [53, p. 7]).

6. "It would be interesting to be notified not just about the AV's intention to stop but also about where it intends to stop." (Marieke Martens, [53, p. 8]).

7. "The design should ensure that users direct their attention to the right information at the right time." (Natasha Merat, [53, p. 9]).

These quotes served as the foundation for designing the concepts during the following phases.

\subsection{Phase 2: Collection of relevant literature}

Literature was collected to serve as a theoretical foundation for the subsequent design phase. For each idea reported above, two authors (Authors 1 and 5) identified supported theories and related concepts from a previously compiled literature folder containing 357 papers on eHMIs, AR, and other supports for VRUs. The selection of papers was based on seminal works in the field of Human Factors and Ergonomics.

\subsection{Phase 3: Brainstorming design sessions}

Brainstorming design sessions were conducted amongst the authors (Authors 1, 2, 4, \& 5). The same scenario with a vehicle approaching from the right was envisaged for all concepts. An unmarked twoway road in Malta was chosen as the environment on which the AR concepts would be mapped. The car was stationary in front of the pedestrian crossing area to allow all AR concepts to be demoed irrespective of technical limitations.

It was agreed that two states would be created per concept, namely, 'vehicle is yielding', and 'vehicle is not yielding'. These states would be communicated using green and red, respectively, consistent with a study that showed these colours to be most intuitive for pedestrians judging the intentions of an approaching vehicle [2]. None of the concepts would communicate instructions, as instructions may lead to erroneous crossing decisions [30]. Moreover, it was decided that concepts that were not augmented on the ground would be made of translucent material not to block the user's view of the real world. It was agreed that each concept had a supporting cue, such as movement or iconography, to facilitate redundancy gain [29].

MIRO [38], an online collaborative environment, was used as a supporting tool. The MIRO board contained an affinity diagram and a table with 15 design principles for human-computer interaction [29]. Images taken from a LiDAR scan of the chosen environment were added to the board to aid the participants.

During the brainstorm, each concept idea from Phase 1 and corresponding literature from Phase 2 was brought forward. Design elements (e.g., dimensions, form, iconography, animation) were discussed at length with reference to the literature identified for the concept (see Phase 2). For each concept, virtual post-it notes 

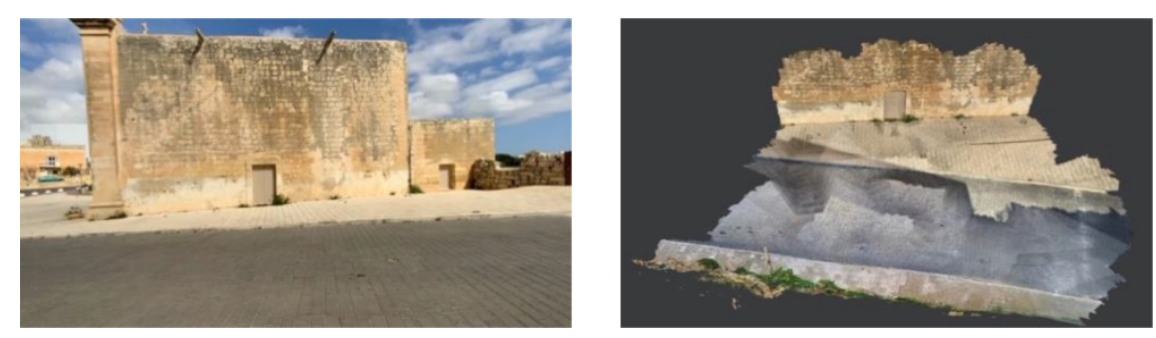

Figure 1: Left: The chosen unmarked crossing in Malta. Right: The captured LiDAR 3D scan on location.

representing different design elements were added to the board. The authors also used experience-based design to reach decisions. In total, the brainstorming was spread across three separate sessions for a total of 5 hours.

Through the design process, the seven design ideas extracted from Tabone et al. [53] evolved into the following nine AR concepts: (1) augmented zebra crossing, (2) planes on vehicle, (3) conspicuous and looming planes, (4) field of safe travel, (5) fixed pedestrian traffic lights, (6) virtual fence, (7) phantom car, (8) nudge HUD, (9) pedestrian traffic lights HUD.

A low-fidelity paper sketch was created for each concept based on the design elements identified for each concept. Finally, a heuristic evaluation was carried out based on nine AR heuristics proposed by Endsley et al. [12]. If shortcomings in the design were flagged through the heuristic evaluation, a design modification was conducted to accommodate. Examples include modifying Concepts 8 and 9 so that the messages were placed at the top of the field of view (FOV) rather than the middle to support the heuristic 'fit with the user's perceptual abilities'. Heuristics that were approved without modification to the concepts include 'alignment of physical and virtual worlds', 'accounting for hardware capabilities', and 'adaptation to user position and motion', amongst others. Once all authors were confident with the modified sketches and heuristic checklist, the concepts were moved to Phase 4 .

\subsection{Phase 4: Implementation}

2.4.1 Hardware and software. The implementation was carried out using Unity 3D and its new tool for AR developers: Mixed and Augmented Reality Studio (MARS) v.1.2, together with Apple ARKit (v.2.1.9) and AR Foundation (v.2.1.8) libraries. A 15" MacBook Pro (2019) with $2.3 \mathrm{GHz}$ 8-Core Intel Core i9 processor, 16 GB DDR4 memory, and Radeon Pro 560X 4 GB GPU was used for development, whereas an iPad Pro 11" (2020) was used as the target device because of its inbuilt LiDAR. A Toyota Yaris Hybrid vehicle was used for demonstrating the AR concepts.

A LiDAR 3D scan of the crossing and surrounding area of the road was captured (Figure 1) using the $3 \mathrm{dScanner}$ app [56] on the iPad set on a lower quality setting to reduce storage requirements. The scan with 1.1 million vertices was exported as an OBJ file and imported into Blender, where texture files extracted from the 3D scanning software were added, and the final scan was exported as an FBX file. A Unity MARS simulation environment was created using the FBX to test the augmented concepts in the Unity editor before actual testing on site.
2.4.2 Implementing the AR concepts in MARS.. To anchor the concepts to the real world, several Unity MARS tools were used [61]. Proxies, representing real-world objects (e.g., a flat surface, vehicle) that the final application could detect, were used to define regions where augmented elements could appear. Some proxies included semantic tags (i.e., to specify that they represent the user in the real world or the floor), whereas others included Conditions, Rules, and Forces, which affect the behaviour of the proxies.

Our implemented app, built through Unity MARS, used the camera and LiDAR of the iPad to scan the real environment, generate planes of the world, and spawn objects if proxy conditions were met (e.g., horizontal surface of a certain size). During the first visit to the testing location, the Unity MARS companion app [62] was used to capture the environment dimensions, image markers, and proxy data of the crossing region and vehicle, to be used in the implementation.

For each implementation, the objects that comprise the AR concept were added as children to their Proxy object. ShowChildrenOnTrackingAction was applied, so that child objects were spawned, oriented, and anchored with the proxy object once the proxy's conditions were met.

For concepts that were augmented on the ground (i.e., Concepts 1 and 6), the crossing region's horizontal proxy data was used together with a number of conditions. More specifically, IsPlaneCondition, FlatFloorCondition, and a 'floor' semantic tag were added to specify that the object to augment upon had to be a flat surface and a floor. An AlignmentCondition was added to align objects in a HorizontalUp orientation, whereas a PlaneSizeCondition specified that a $1.5 \mathrm{~m} \times 1.5 \mathrm{~m}$ flat plane had to be scanned and mapped for augmentation to occur. To make the AR concept align with the user, the objects augmented on the proxy were aligned to a ProxyObjectReferencePerson (representing the user's position) through a ProxyAlignmentForce.

Image markers (i.e., doorway across the street, photo of the car) were used for instances where a crossing region (i.e., Concepts 5 and 7) or a car (i.e., Concepts 2 and 3) had to be recognised. In these cases, a MarkerCondition was added to the proxy, and the image to be recognised was selected from the Marker Library imported through the companion app. The child objects would spawn and anchor to the real-world region that was mapped through the image marker.

For Concept 4, a direct placement script by the Unity MARS team [60] was used so that an object could be spawned and mapped to any surface found by raycasting. Lastly, concepts with a HUD 

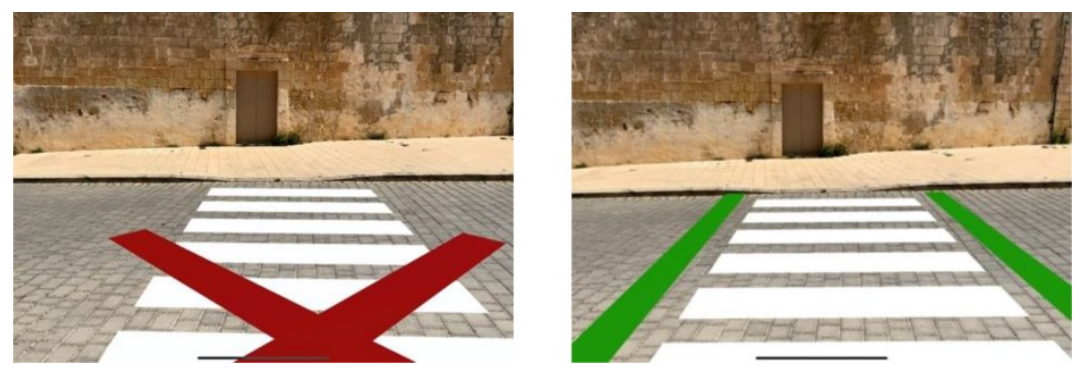

Figure 2: Concept 1. Left: non-yielding state with an ' $\mathrm{X}$ ' placed on top of the augmented zebra crossing. Right: yielding state with two green bars flanking the zebra crossing.

(Concepts 8 and 9) used the traditional Unity UI workflow in a Unity MARS session.

2.4.3 Creating the final application and testing on-site. For each concept, a plane or point cloud visualisers were added to offer visual feedback to the user that the system was detecting the environment during operation. The shifting from the yielding to the non-yielding state was performed manually via a tap on the iPad screen.

During each iteration, the prototype was built as an iOS app onto the iPad and tested at the chosen location until its performance concerning the mapping of the visual elements to the real world was satisfactory. A single app comprising all implemented concepts and a menu was exported and executed on-site. The prototypes were recorded using the in-built iOS screen capture. These recordings, together with an additional number of demos involving a moving car, which yielded or maintained speed, were compiled into a single video, which is available as supplementary material. In addition, the material includes a photograph detailing how the iPad was used.

\section{RESULTS AND DISCUSSION}

\subsection{Concept 1: Augmented zebra crossing}

Concepts 1 and 2 were derived from the first quote in Phase 1. For Concept 1, the part on "providing a unified affordance rather than just a visual colouring" was taken into account for the road. Hence, for Concept 1, we decided to change the surface and colour of the road (Figure 2). Similar non-augmented concepts have used LEDs embedded in the road to display crossings on-demand [59]. In the same vein, Eriksson et al. [13] presented an in-vehicle augmented HMI that showed a red demarcation or green carpet on the motorway depending on whether it was unsafe or safe to change lanes.

Our prototype for Concept 1 in a non-yielding state consists of a zebra crossing with a red cross overlaid on top. For the yielding state, the red cross is replaced by two rectangular green bars that flank the zebra crossing on either vertical side. These display elements were inspired by a previously published implementation of a smart road [33]. A zebra crossing with additional elements was preferred over an entire green/red surface with overlaid arrows because the latter may offer an instructive suggestion to cross.

\subsection{Concept 2: Planes on vehicle}

This AR concept entailed a change of the vehicle surface (Figure 3 ). We added a plane that would appear at the top front part of the vehicle. While vehicle kinematics combined with such an AR concept may already offer a rich cue, there is still the issue that a red car may be perceived as yielding (rather than the green car) $[2,30]$. Therefore, redundancy gain was employed [65] by superimposing standard icons representing yielding (walking human figure) and non-yielding (hand palm) on the windshield, considering that pedestrians are likely to focus their visual attention on that region $[9,66]$.

In our prototype and supplementary video, the non-yielding red plane is spawned as soon as the image marker condition for the vehicle is satisfied (that is, the vehicle used for the image marker is detected).

\subsection{Concept 3: Conspicuous looming planes}

For the third AR concept, which is based on the second quote from Phase 1, the theories of looming and conspicuity [21] were drawn upon. In the study of perception, looming concerns the human response to the rapid approach of a solid body [50]. Through looming, it is possible to communicate threat and distinguish an approaching object from one that is stationary or receding $[6,18]$. Conspicuity is defined as "something that is obvious to the eye or mind, or something that is striking and attracts attention" [37]. Using these principles, Concept 2 was modified so that the red plane (non-yielding) grows in size as it approaches the user and fills the entire FOV by the end, while conversely, the green plane (yielding) shrinks as the vehicle approaches. Hence, threat is mapped to the size of the plane as the vehicle approaches the crossing. In the supplementary video, the camera moves towards the vehicle as the planes grow or shrink, dependent on the state, to simulate the vehicle approaching.

\subsection{Concept 4: Field of safe travel}

The point of departure for this AR concept (Figure 4) was a 'safety corridor' presented to the pedestrian, as mentioned in the third quote from Phase 1. Gibson's field of safe travel [19] was the theory behind this concept. The field at any given moment represents the paths that a car may take unimpeded, and it would look like a "sort of tongue protruding forward along the road" [19,27]. The concept relates to the theory of affordances [17] since the shape of the field is not based on arbitrary rules but reflects physical laws. In simpler terms, the projected field indicates where a vehicle may be in a given amount of time. Hence, if a person enters a red zone, they risk being hit by the vehicle. For the non-yielding state, the red 

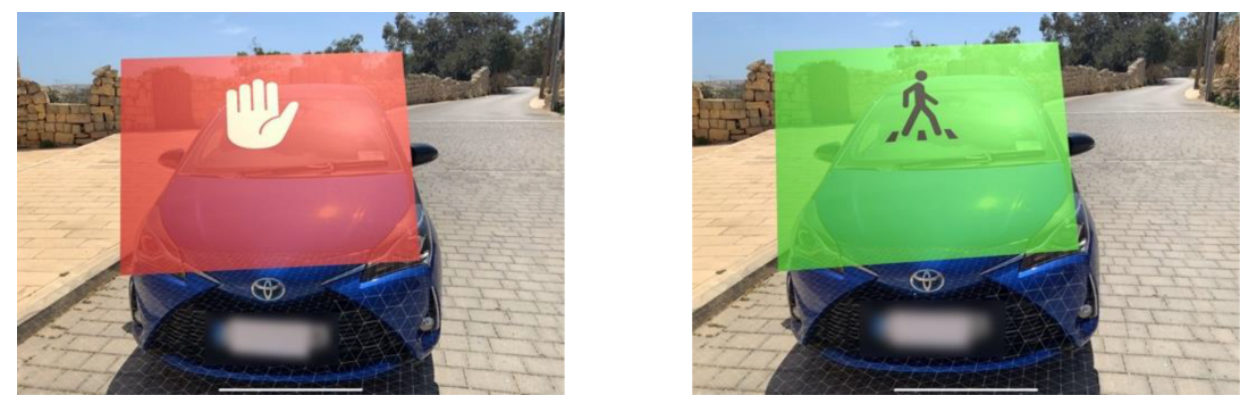

Figure 3: Concept 2. Left: non-yielding state with the red plane and hand icon augmented on the vehicle. Right: yielding state with the green plane and crossing figure icon augmented on the vehicle.
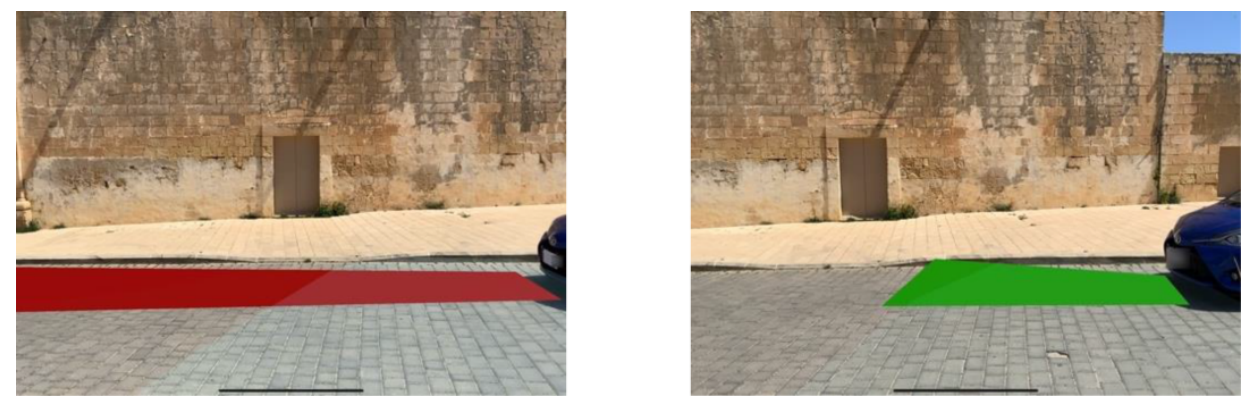

Figure 4: Concept 4. Left: non-yielding state with the red field of safe travel extending over the crossing area. Right: yielding state with the green field of travel shortened and truncating before the crossing area.
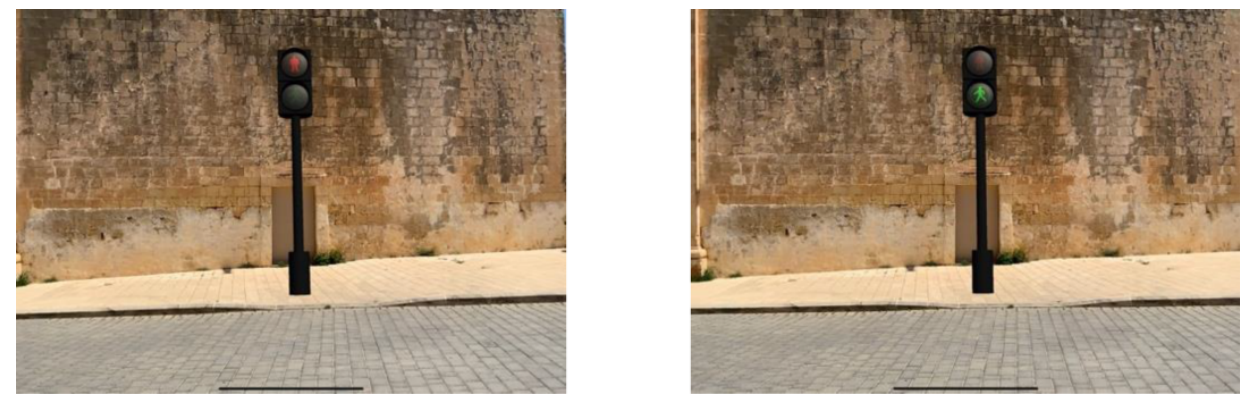

Figure 5: Concept 5. Left: non-yielding state with red pedestrian icon. Right: yielding state with green pedestrian icon.

field extends beyond the crossing point, whereas for the yielding state, the field switches to green, widens, and terminates prior to the point of crossing to emphasize the vehicle will not cross beyond this point.

In our approach, the field is represented as a flat plane emerging from the front of the vehicle. If the prototype was to be fully implemented, the plane's length would be proportional to the vehicle's speed. However, to demonstrate both states using the prototype for the supplementary video, the length of the plane was hardcoded. Hesenius et al. [23] presented a similar concept of Safe Zones for pedestrians, highlighting regions on the road. Areas marked in green denoted zones that could be safely traversed; conversely, areas marked in red denoted potentially dangerous areas to navigate.

\subsection{Concept 5: Fixed pedestrian traffic lights}

For this AR concept (Figure 5), based on the fourth quote in Phase 1, the familiar concept of binary pedestrian traffic lights was chosen. These lights would pop up on the other side of the crossing area to alert the pedestrian whether a vehicle is approaching and intending to yield. As an additional cue to the red and green traffic light beams used for the non-yielding and yielding states, icons of a static and walking human figure are superimposed, respectively. Moreover, since this pedestrian traffic light is a replica of its realworld counterpart, it is attached to the ground to comply with the user's mental model.

For the implementation, the 3D model of the lights was set as a child of the crossing image marker proxy. Once the iPad was 

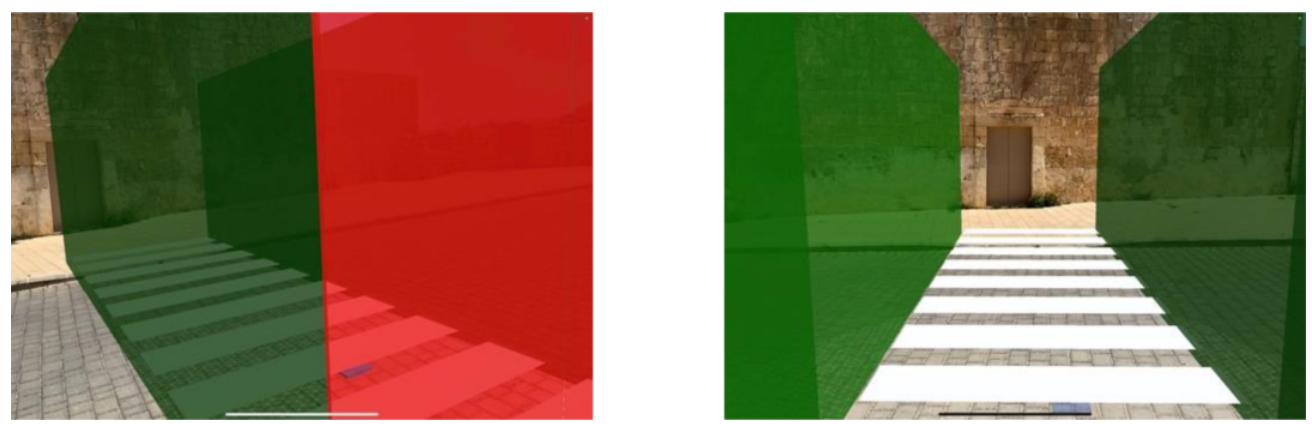

Figure 6: Concept 6. Left: non-yielding state with the closed red gate 'blocking' the pedestrian. Right: yielding state, where the gate is green and opened.

pointed towards the doorway across the road, the traffic lights model was augmented on the pavement.

\subsection{Concept 6: Virtual fence}

Concept 6 was to "augment a 3D traffic light in the form of a virtual fence", as stated in the fifth quote from Phase 1. Discussions commenced with the idea of having a fence preventing pedestrians from entering the road. However, concerns arose that the fence could invite the pedestrian to walk around it, which is undesirable. Therefore, the fence concept was operationalized in the form of a tunnel (Figure 6).

The tunnel is created using semi-translucent green walls on either side of the crossing area, high enough to enclose a person with an average height. A zebra crossing is displayed on the tunnel's floor to offer redundancy gain, while a semi-translucent red gate of the same height blocks the pedestrian's path to the other side of the road in the non-yielding state. The sides of the tunnel are green to indicate that cars could cross through that side of the tunnel. The gate's colour switches to green for the yielding state, and an animation of the gate swinging open plays out. For the implementation, the proxies and zebra crossing used in Concept 1 were built upon.

The 'tunnels in the sky' $[41,58]$ AR/HUD concept from the domain of aviation was a source of inspiration for the design. Furthermore, a 'safe corridor' augmented in-vehicle HMI, which communicates to the driver whether an area on the road should be avoided (if red) or safely steered into (green) [34], served as an additional source of inspiration. It has similarities to the on-the-road carpet HMI consulted for Concept 1 [13]. These carpet metaphors inspired the addition of a zebra crossing placed on the ground between the walls.

\subsection{Concept 7: Phantom car}

In this case, in addition to the vehicle's intention to yield, the concept communicates its intended stopping location (Figure 7). A previously published concept called 'Vehicle Intents' [23] highlighted the vehicle's path with an arrow and added stopping lines to communicate where the vehicle would stop if the pedestrian was to cross. The idea was further developed to include an augmented representation of a hatchback car, similar to what could be seen as part of a ghost replay system in a racing computer game or Tesla's shadow mode [14].

Hence, the phantom car concept was created. A semi-translucent vehicle, which switches between green and red depending on the scenario, is augmented. The phantom car moves in front of the real vehicle to indicate where the vehicle would be after a particular time (e.g., 2 seconds into the future). In the yielding scenario, as the real vehicle slows down, the distance between itself and the green phantom car will shrink until the real vehicle has fully entered the phantom car. In the non-yielding scenario, the red phantom car will communicate its intent by moving in front of the actual vehicle at a constant distance.

In the prototype and supplementary video, the non-yielding red phantom car was augmented on the crossing to simulate that the vehicle intends to move beyond the stopping area; hence, it is unsafe to cross. In the yielding scenario, the green phantom car was augmented next to the stationary real vehicle to simulate the moment before the real vehicle had fully entered the area occupied by the phantom car.

\subsection{Concept 8: Nudge HUD}

Distracted pedestrians are the cause of many accidents [1, 42]. An interface that assists the pedestrian with looking towards the right direction may therefore be beneficial. This concept addresses a different challenge from the previous concepts, as the latter assume that the pedestrian is looking at the AR display anchored to the environment. In Concept 8, a HUD that follows the pedestrian's FOV regardless of where they are looking, was created (Figure 8).

The HUD was drawn in a billboard style where text in sans serif typeface Arial was placed on a background plane of a different colour to offer good performance in terms of readability [3, 16, 47]. For the non-yielding state, the text is "DANGER! VEHICLE IS APPROACHING", which changes to "SAFE TO CROSS" in the yielding state [15]. The messages use non-instructing language since there are dangers of misinterpretation in providing explicit advice [30]. Icons were added to the left of the text and switched between an outstretched hand and a walking human figure, respectively.

The HUD was display-locked so that it stayed in the same position on the display, which would be analogous to head-locked positioning on a head-mounted display such as AR glasses. This placement decision was based on empirical research recommending 

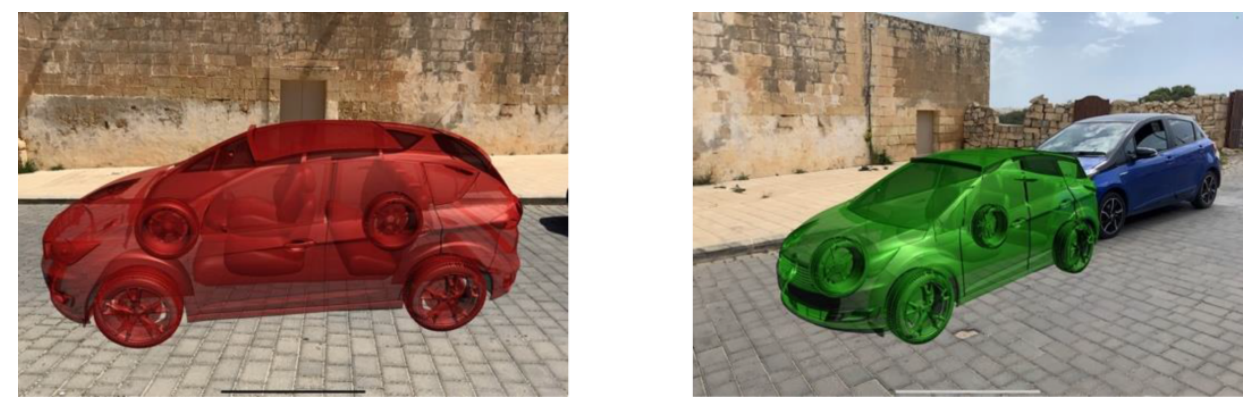

Figure 7: Concept 7. Left: non-yielding state with the red phantom car blocking the crossing area. Right: yielding state with the real car entering the green phantom car.
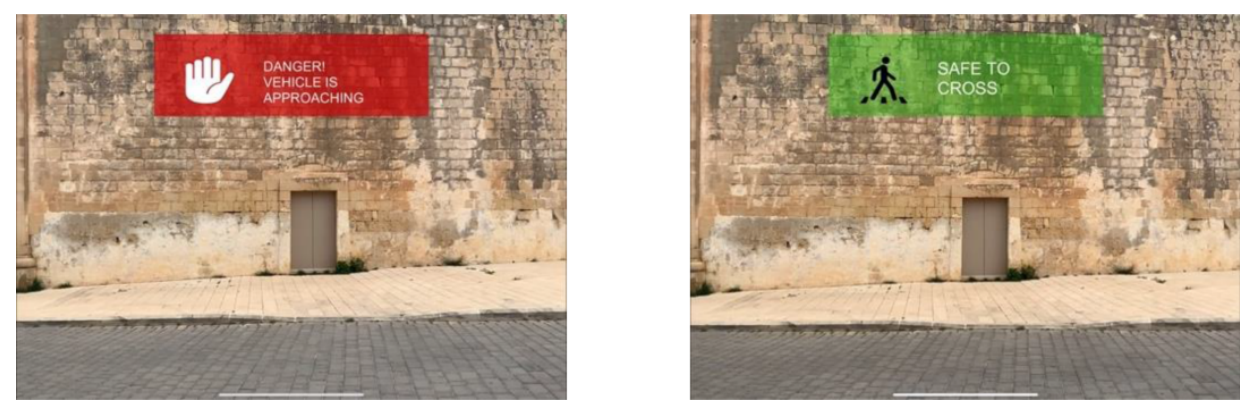

Figure 8: Concept 8. Left: the non-yielding state with the red HUD showing the hand icon and corresponding text message. Right: the yielding state with the green HUD, walking pedestrian icon, and corresponding text message.
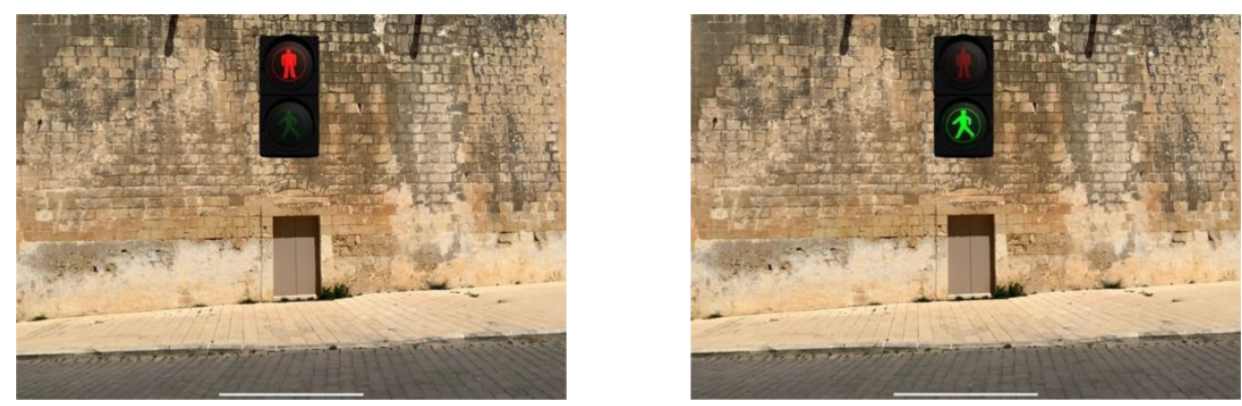

Figure 9: Concept 9. Left: non-yielding state with the red pedestrian icon. Right: yielding state with the green pedestrian icon.

head-locked text placement if the real-world task involves permanent visual monitoring in the central or near-peripheral visual field [26], which in our case would be monitoring of the vehicle. Moreover, the HUD was placed in the centre top section of the FOV since it is recommended to place text in top positions in complex environments that require constant monitoring [26]. Top positioning would also leave the bottom part of the FOV not occluded so that the pedestrian could still see where they are walking.

\subsection{Concept 9: Pedestrian traffic lights HUD}

For this concept, the pedestrian traffic light model used for Concept 5 is displayed as a HUD that follows the user's FOV (Figure 9). The model was modified to remove the pole that attaches the traffic light to the ground to create a 'detached' interface element. Moreover, the same interface placement location used for Concept 8 was adopted, with the difference that instead of using UI elements, the 3D traffic light model was made a child of the main camera to follow the FOV.

\section{GENERAL DISCUSSION}

In this paper, nine AR concepts that aim to support pedestrians in making crossing decisions when encountering future traffic in an urban environment were presented as implemented prototypes. Expert perspectives [53] were analysed to extract AR concept ideas. Subsequently, a literature analysis was performed to identify similar approaches and theories that aided in transforming the idea into a prototype. Following this phase, brainstorming design sessions were conducted, resulting in nine designs in the form of sketches on paper. Underlying these ideas were theories of affordances [17], 
Table 1: Sensor requirements for the concepts.

\begin{tabular}{lllll}
\hline Concept & Road position & Vehicle intent & Vehicle position & Vehicle speed \\
\hline (1) Augmented zebra crossing & $\mathrm{X}$ & $\mathrm{X}$ & $\mathrm{X}$ & \\
(2) Planes on vehicle & & $\mathrm{X}$ & $\mathrm{X}$ & $\mathrm{X}$ \\
(3) Conspicuous looming planes & $\mathrm{X}$ & $\mathrm{X}$ & $\mathrm{X}$ \\
(4) Field of safe travel & $\mathrm{X}$ & $\mathrm{X}$ & $\mathrm{X}$ \\
(5) Fixed pedestrian traffic lights & $\mathrm{X}$ & $\mathrm{X}$ & $\mathrm{X}$ & \\
(6) Virtual fence & $\mathrm{X}$ & $\mathrm{X}$ & $\mathrm{X}$ \\
(7) Phantom car & & $\mathrm{X}$ & \\
(8) Nudge HUD & & $\mathrm{X}$ & \\
(9) Pedestrian traffic lights HUD & & & \\
\hline
\end{tabular}

multimodal communication [5], looming and conspicuity [21, 50], and AR research in aerospace $[41,58]$. Finally, implementation was conducted using Unity MARS, a new tool for AR developers, and the AR concepts were tested and filmed at an uncontrolled crossing.

During the brainstorming process and based on the literature consulted, it was decided that colour alone was 'not enough' and that redundancy gain had to be employed [65]. Therefore, icons were added. In the same vein, the mere presence of a zebra crossing does not guarantee safety in the real world. With the addition of the red cross and the green bars, the interface communicated that it was unsafe or safe to cross at that moment. Moreover, based on the literature, it was decided that messages should not be instructive [30]. Hence, the concepts were made comparable in the sense that none instructed pedestrians but rather gave the suggestion that it is safe or unsafe to cross.

Occlusion $[39,67]$ was a difficult factor to handle, which is why this was discussed in detail during the brainstorming session and the subsequent heuristic evaluation. The occlusion issue [44] was experienced first-hand, where the gate and walls of the virtual fence (Concept 6) had to be semi-translucent so that the vehicle could be seen. The same principle was applied to the other concepts that were not augmented on the road surface.

\subsection{Limitations and future work}

Several assumptions were made, and the presented concepts should not be viewed as fully finished AR interfaces that VRUs can already use. The implemented concepts and videos take only one vehicle into account. Furthermore, although most of the prototypes were augmented using Unity MARS functionalities such as Proxies, Conditions, and Forces, for all concepts, the state change for vehicle intent (i.e., yielding, non-yielding) was manually triggered using a tap on the iPad. In real-life applications, the path planner of the $\mathrm{AV}$, connected to its $\mathrm{CAN}$ bus, could send out a wireless signal to the VRU's AR system when the AV-VRU distance or time-to-arrival drops below a threshold value.

Concepts such as 8 and 9 were easy to implement as little context is needed for these to operate. However, because of the nascent status of the technology used, various workarounds were implemented for concepts that required knowledge of the position of the approaching vehicle (Concepts 2, 3, 4, 7) and its speed (Concepts 3,4 , and 7). These workarounds included having the real vehicle remaining static since moving real-world objects are not yet supported by the libraries used. While the road position was handled by Unity MARS through the camera and LiDAR, vehicle position was hardcoded, or image markers were relied upon. Moreover, the dimensions of the zebra crossing and tunnel for Concepts 1 and 6 were set manually, based on the 3D LiDAR scan model of the environment. For Concept 3, vehicle position readouts from LiDAR raycasts were tried to map the looming plane's size with AV-pedestrian distance. However, the desired precision was not attained, and hence an animator script was used to resize the planes. Future improvements could be accomplished through edge computer vision methods to detect the distance and speeds of approaching road users. Alternatively, IoT communication could be used, where AVs transmit their yielding intent, position, and speed wirelessly to the VRU, as pointed out above.

Although the current implementation was done on an iPad Pro (to make use of its LiDAR), the concepts could be readily exported to the head-mounted displays currently supported by Unity and Unity MARS. In that case, sensor requirements would need to be met, and issues such as outdoor luminance levels that may hinder perception [11], ocular vergence-accommodation conflicts [48, 64] and latency issues leading to visually induced motion sickness [4] have to be considered. Sensor requirements vary between concepts according to complexity, as shown in Table 1.

In the future, user studies are needed to identify which concepts are best accepted, how the concepts would function on AR glasses in the urban environment, and what triggers should be used to change between yielding and non-yielding states. The intuitiveness of the mappings used, such as the intuitiveness of the looming cues in Concept 3 and the red vs. green colours used for the tunnel walls in Concept 6, needs to be investigated as well. Furthermore, it would be important to include provisions for VRUs with visual impairments, for example, by adding tactile or auditory signals. Finally, it would be worthwhile to explore how to integrate the AR concepts with other communication modalities for VRUs, such as eHMIs and smart infrastructure.

\section{CONCLUSION}

In conclusion, this paper presents an outlook on future AV-VRU communication through AR in the form of prototypes. This paper presents nine such concepts designed using theory, expert opinion, 
literature, brainstorm, and implementation in Unity. In the spirit of open science, the code is provided as supplementary material to encourage further development.

\section{ACKNOWLEDGMENTS}

The authors would like to acknowledge the Unity MARS team for their generous support.

This project has received funding from the European Union's Horizon 2020 research and innovation programme under the Marie Skłodowska-Curie grant agreement No 860410.

\section{REFERENCES}

[1] Benjamin K. Barton, Susan M. Kologi, and Anne Siron. 2016. Distracted pedestrians in crosswalks: An application of the Theory of Planned Behavior. Transportation Research Part F: Traffic Psychology and Behaviour 37 (Feb. 2016), 129-137. DOI: https://doi.org/10.1016/j.trf.2015.12.012

[2] Pavlo Bazilinskyy, Dimitra Dodou, and Joost De Winter. 2020. External HumanMachine Interfaces: Which of 729 colors is best for signaling 'Please (do not) cross'? In 2020 IEEE International Conference on Systems, Man, and Cybernetics $(S M C)$, October 11 - 14, 2020, Toronto, ON, Canada, 3721-3728. DOI: https://doi. org/10.1109/SMC42975.2020.9282998

[3] Michael L. Bernard, Barbara S. Chaparro, Melissa M. Mills, and Charles G. Halcomb. 2003. Comparing the effects of text size and format on the readibility of computer-displayed Times New Roman and Arial text. International Journal of Human-Computer Studies 59, 6 (Dec. 2003), 823-835. DOI: https: //doi.org/10.1016/S1071-5819(03)00121-6

[4] Timothy J. Buker, Dennis A. Vincenzi, and John E. Deaton. 2012. The effect of apparent latency on simulator sickness while using a see-through helmet-mounted display: Reducing apparent latency with predictive compensation. Human Factors 54, 2 (Jan. 2012), 235-249. DOI: https://doi.org/10.1177/0018720811428734

[5] Jennifer L. Burke, Robin R. Murphy, Erika Rogers, Vladimir J. Lumelsky, and Jean Scholtz. 2004. Final report for the DARPA/NSF interdisciplinary study on human-robot interaction. IEEE Transactions on Systems, Man, and Cybernetics, Part C (Applications and Reviews) 34, 2 (May 2004), 103-112. DOI: https://doi.org/ 10.1109/TSMCC.2004.826287

[6] Jeff K. Caird and Peter A. Hancock. 1994. The perception of arrival time for different oncoming vehicles at an intersection. Ecological Psychology 6, 2 (1994), 83-109. DOI: https://doi.org/10.1207/s15326969eco0602_1

[7] Koen de Clercq, Andre Dietrich, Juan P. N. Velasco, and Joost de Winter, 2019 External Human-Machine Interfaces on automated vehicles: effects on pedestrian crossing decisions. Human Factors 61, 8 (Mar. 2019), 1353-1370. DOI: https://doi. org/10.1177/0018720819836343

[8] Debargha Dey, Azra Habibovic, Andreas Löcken, Philipp Wintersberger, Bastian Pfleging, Andreas Riener, Marieke Martens, and Jacques Terken, 2020. Taming the eHMI jungle: A classification taxonomy to guide, compare, and assess the design principles of automated vehicles' external human-machine interfaces, Transportation Research Interdisciplinary Perspectives, 7, 9, (Sep. 2020), 100174. DOI: https://doi.org/10.1016/j.trip.2020.100174

[9] Debargha Dey, Francesco Walker, Marieke Martens, and Jacques Terken. 2019 Gaze patterns in pedestrian interaction with vehicles: Towards effective design of external Human-Machine Interfaces for automated vehicles. In Proceedings of the 11th International Conference on Automotive User Interfaces and Interactive Vehicular Applications (AutomotiveUI '19). Association for Computing Machinery, New York, NY, USA, 369-378. DOI: https://doi.org/10.1145/3342197.3344523

[10] André Dietrich, Jan-Henning Willrodt, Karolin Wagner, and Klaus Bengler. 2019. Projection-based external human machine interfaces-enabling interaction between automated vehicles and pedestrians. In Proceedings of the DSC 2018 Europe $V R$, September 5 - 7, 2018, Antibes, France, 43-50.

[11] Tiffany D. Do, Joseph J. LaViola, and Ryan P. McMahan. 2020. The effects of object shape, fidelity, color, and luminance on depth perception in handheld mobile augmented reality. In 2020 IEEE International Symposium on Mixed and Augmented Reality (ISMAR), November 9 - 13, Porto de Galinhas, Brazil, 64-72. DOI: https://doi.org/10.1109/ISMAR50242.2020.00026

[12] Tristan C. Endsley, Kelly A. Sprehn, Ryan M. Brill, Kimberly J. Ryan, Emily C. Vincent, and James M. Martin. 2017. Augmented reality design heuristics Designing for dynamic interactions. In Proceedings of the Human Factors and Ergonomics Society Annual Meeting 61, 1, (Oct. 2017), Sage California, Los Angeles, California, 2100-2104. DOI: https://doi.org/10.1177/1541931213602007

[13] Alexander Eriksson, Sebastiaan M. Petermeijer, Markus Zimmermann, Joos De Winter, Klaus J. Bengler, and Neville A. Stanton. 2018. Rolling out the red (and green) carpet: supporting driver decision making in automation-to-manual transitions. IEEE Transactions on Human-Machine Systems 49, 1 (Feb. 2019), 20-31. DOI: https://doi.org/10.1109/THMS.2018.2883862
[14] Forbes. 2019. Tesla's "Shadow" Testing Offers A Useful Advantage On The Biggest Problem In Robocars. (April 2019). Retrieved March 25, 2021 from https://www.forbes.com/sites/bradtempleton/2019/04/29/teslas- shadowtesting-offers-a-useful-advantage-on-the-biggest-problem-in-robocars

[15] Lex Fridman, Bruce Mehler, Lei Xia, Yangyang Yang, Laura Yvonne Facusse, and Bryan Reimer. 2017. To walk or not to walk: Crowdsourced assessment of external vehicle-to-pedestrian displays. arXiv:1707.02698. Retrieved from https: //arxiv.org/abs/1707.02698

[16] Joseph L. Gabbard, J. Edward Swan, and Deborah Hix. 2006. The effects of text drawing styles, background textures, and natural lighting on text legibility in outdoor augmented reality. Presence: Teleoperators \& Virtual Environments 15, 1 (Feb. 2006), 16-32. DOI: https://doi.org/10.1162/pres.2006.15.1.16

[17] James J. Gibson. 1986. The Ecological Approach to Visual Perception (Vol. 1). Psychology Press, New York.

[18] James J. Gibson and Leonard Carmichael. 1966. The Senses Considered as Perceptual Systems (Vol. 2, No. 1). Houghton Mifflin, Boston.

[19] James J. Gibson and Laurence E. Crooks. 1938. A theoretical field-analysis of automobile-driving. The American fournal of Psychology 51, 3 (Jul. 1938), 453-471. DOI: https://doi.org/10.2307/1416145

[20] Jens Grubert, Tobias Langlotz, Stefanie Zollmann, and Holger Regenbrecht. 2016. Towards pervasive augmented reality: Context-awareness in augmented reality. IEEE Transactions on Visualization and Computer Graphics 23, 6 (Mar. 2016), 17061724. DOI: https://doi.org/10.1109/TVCG.2016.2543720

[21] Peter A. Hancock. 2019. On the dynamics of conspicuity. Human Factors 61, 6 (April 2019), 857-865. DOI: https://doi.org/10.1177/0018720819840849

[22] Michael Hartmann, Marco Viehweger, Michael Stolz, Daniel Watzenig, Michael Spitzer, and Wim Desmet. 2018. "Pedestrian in the Loop": An approach using augmented reality. SAE Technical Paper, 2018-01-1053. DOI: https://doi.org/10. 4271/2018-01-1053

[23] Marc Hesenius, Ingo Börsting, Ole Meyer, and Volker Gruhn. 2018. Don’t panic! Guiding pedestrians in autonomous traffic with augmented reality. In Proceedings of the 20th International Conference on Human-Computer Interaction with Mobile Devices and Services Adjunct (MobileHCI '18). Association for Computing Machinery, New York, NY, USA, 261-268. DOI: https://doi.org/10.1145/3236112.3236148

[24] Stewart Von Itzstein, Mark Billinghurst, Ross T. Smith, and Bruce H. Thomas. 2017. Augmented reality entertainment: Taking gaming out of the box. In Encyclopedia of Computer Graphics and Games. Springer International Publishing, Cham, 1-9. DOI: https://doi.org/10.1007/978-3-319-08234-9_81-1

[25] Vinu Kamalasanan, Frederik Schewe, Monika Sester, and Mark Vollrath. 2021. Exploratory Study on the Use of Augmentation for Behavioural Control in Shared Spaces. (Jul 2021). In Proceedings of the $13^{\text {th }}$ International Conference on Virtual, Augmented and Mixed Reality (VAMR 2021). Springer, Cham, 18-31. DOI: https: //doi.org/10.1007/978-3-030-77599-5 2

[26] Elisa M. Klose, Nils Adrian Mack, Jens Hegenberg, and Ludger Schmidt. 2019. Text presentation for augmented reality applications in dual-task situations. In 2019 IEEE Conference on Virtual Reality and 3D User Interfaces (VR), March 23 27, 2019, Osaka, Japan, 636-644. DOI: https://doi.org/10.1109/VR.2019.8797992

[27] Sarvesh Kolekar, Joost de Winter, and David Abbink. 2020. Human-like driving behaviour emerges from a risk-based driver model. Nature Communications 11, 1 (Sep. 2020), 1-13. DOI: https://doi.org/10.1038/s41467-020-18353-4

[28] Aymen Lakehal, Sophie Lepreux, Christos Efstratiou, Christophe Kolski, and Pavlos Nicolaou. 2020. Investigating smartphones and AR glasses for pedestrian navigation and their effects in spatial knowledge acquisition. In 22nd International Conference on Human-Computer Interaction with Mobile Devices and Services (MobileHCI '20). Association for Computing Machinery, New York, NY, USA, Article 11, 1-7. DOI: https://doi.org/10.1145/3406324.3410722

[29] John D. Lee, Christopher D. Wickens, Yili Liu, and Linda N. Boyle. 2017. Designing for People. CreateSpace.

[30] Yee Mun Lee, Ruth Madigan, Jorge Garcia, Andrew Tomlinson, Albert Solernou, Richard Romano, Gustav Markkula, Natasha Merat, and Jim Uttley. 2019. Understanding the messages conveyed by automated vehicles. In Proceedings of the 11th International Conference on Automotive User Interfaces and Interactive Vehicular Applications (AutomotiveUI '19). Association for Computing Machinery, New York, NY, USA, 134-143. DOI: https://doi.org/10.1145/3342197.3344546

[31] Yee Mun Lee, Ruth Madigan, Chinebuli Uzondu, Jorge Garcia, Richard Romano, Gustav Markkula, and Natasha Merat. 2020. Learning to interpret novel eHMI: The effect of vehicle kinematics and eHMI familiarity on pedestrians' crossing behaviour. Retrieved from https://psyarxiv.com/2xub4

[32] Mark A. Livingston, Lawrence J. Rosenblum, Dennis G. Brown, Gregory S. Schmidt, Simon J. Julier, Yohan Baillot, J. Edward SwanII, Zhuming Ai, and Paul Maassel. 2011. Military applications of augmented reality. In Handbook of augmented reality. Springer, New York, NY, 671-706. DOI: https://doi.org/10.1007/9781-4614-0064-6_31

[33] Andreas Löcken, Carmen Golling, and Andreas Riener. 2019. How should automated vehicles interact with pedestrians? A comparative analysis of interaction concepts in virtual reality. In Proceedings of the 11th International Conference on Automotive User Interfaces and Interactive Vehicular Applications (AutomotiveUI 
'19). Association for Computing Machinery, New York, NY, USA, 262-274. DOI: https://doi.org/10.1145/3342197.3344544

[34] Lutz Lorenz, Philipp Kerschbaum, and Josef Schumann. 2014. Designing take over scenarios for automated driving: How does augmented reality support the driver to get back into the loop?. In Proceedings of the Human Factors and Ergonomics Society Annual Meeting 58, 1, (Oct. 2014), Sage California, Los Angeles, California, 1681-1685. DOI: https://doi.org/10.1177/1541931214581351

[35] Dengzhe Ma, Jügen Gausemeier, Xiumin Fan, and Michael Grafe. 2011. Virtual Reality \& Augmented Reality in Industry. Springer, Berlin Heidelberg.

[36] Karthik Mahadevan, Sowmya Somanath, and Ehud Sharlin. 2018. Communicating awareness and intent in autonomous vehicle-pedestrian Interaction. In Proceedings of the 2018 CHI Conference on Human Factors in Computing Systems (CHI '18). Association for Computing Machinery, New York, NY, USA, Paper 429, 1-12. DOI: https://doi.org/10.1145/3173574.3174003

[37] Merriam-Webster. 2021. Dictionary by Merriam-Webster. (October 2017). Retrieved March 25, 2021 from https://www.merriam-webster.com

[38] Miro. 2021. An Online Visual Collaborative Platform for Teamwork. (March 2021) Retrieved March 25, 2021 from https://miro.com/.

[39] Manisah Mohd Shah, Haslina Arshad, and Riza Sulaiman. 2012. Occlusion in augmented reality. In 8th International Conference on Information Science and Digital Content Technology (ICIDT2012), June 26 - 28, Jeju, South Korea, 372-378.

[40] Angélique Montuwy, Béatrice Cahour, and Aurélie Dommes. 2018. Older pedestrians navigating with AR glasses and bone conduction headset. In Extended Abstracts of the 2018 CHI Conference on Human Factors in Computing Systems (CHI EA '18). Association for Computing Machinery, New York, NY, USA, Paper LBW590, 1-6. DOI: https://doi.org/10.1145/3170427.3188503

[41] Max Mulder. 1999. Cybernetics of Tunnel-In-The-Sky Displays. Ph.D. Dissertation. Delft University of Technology, Delft, The Netherlands. ISBN: 90-407-1963-2.

[42] Judith Mwakalonge, Saidi Siuhi, and Jamario White. 2015. Distracted walking: Examining the extent to pedestrian safety problems. Fournal of Traffic and Transportation Engineering (English edition) 2, 5 (Oct. 2015), 327-337. DOI: https://doi.org/10.1016/j.jtte.2015.08.004

[43] Jacob Nielsen. 1994. Usability Engineering. Morgan Kaufmann, Burlington, Massachusetts.

[44] Jon Peddie. 2017. Augmented Reality: Where We Will All Live. Springer.

[45] Gabriele Pratticò, Fabrizio Lamberti, Alberto Cannavò, Lia Morra, and Paolo Montuschi. 2021. Comparing state-of-the-art and emerging augmented reality interfaces for autonomous vehicle-to-pedestrian communication. IEEE Transactions on Vehicular Technology 70, 2 (Feb. 2021), 1157-1168. DOI: https://doi.org/10. 1109/TVT.2021.3054312

[46] Amir Rasouli and John K. Tsotsos. 2019. Autonomous vehicles that interact with pedestrians: A survey of theory and practice. IEEE Transactions on Intelligent Transportation Systems 21, 3 (Mar. 2019), 900-918. DOI: https://doi.org/10.1109/ TITS.2019.2901817

[47] Helge Renkewitz, Verena Kinder, Mario Brandt, and Thomas Alexander. 2008 Optimal font size for head-mounted-displays in outdoor applications. In 12th International Conference Information Visualisation, July 9 - 11 July, 2008, London, UK, 503-508. DOI: https://doi.org/10.1109/IV.2008.73

[48] Jannick P. Rolland, William Gibson, and Dan Ariely. 1995. Towards quantifying depth and size perception in virtual environments. Presence: Teleoperators \& Virtual Environments 4, 1 (Feb. 1995), 24-49. DOI: https://doi.org/10.1162/pres. 1995.4.1.24

[49] Michelle L. Rusch, Mark C. Schall Jr, John D. Lee, Jeffrey D. 2014. Augmented reality cues to assist older drivers with gap estimation for left-turns. Accident Analysis \& Prevention 71, (Oct. 2014), 210-221. DOI: https://doi.org/10.1016/j.aap. 2014.05.020

[50] William Schiff, James A. Caviness, and James J. Gibson. 1962. Persistent fear responses in rhesus monkeys to the optical stimulus of "looming". Science 136, 3520 (Jun. 1962), 982-983. DOI: https://doi.org/10.1126/science.136.3520.982

[51] Lenja Sorokin, Ronee Chadowitz, and Nina Kauffmann. 2019. A change of perspective: Designing the automated vehicle as a new social actor in a public space.
In Extended Abstracts of the 2019 CHI Conference on Human Factors in Computing Systems(CHI EA '19). Association for Computing Machinery, New York, NY, USA, Paper CS18, 1-8. DOI: https://doi.org/10.1145/3290607.3299044

[52] Wilbert Tabone. 2020. The effectiveness of an augmented reality guiding system in an art museum. In Rediscovering Heritage Through Technology. Springer International Publishing, Cham, 197-214. DOI: https://doi.org/10.1007/978-3-03036107-5_10

[53] Wilbert Tabone, Joost De Winter, Claudia Ackermann, Jonas Bärgman, Martin Baumann, Shuchisnigdha Deb, Colleen Emmenegger, Azra Habibovic, Marjan Hagenzieker, Peter A. Hancock, Riender Happee, Josef Krems, John D. Lee, Marieke Martens, Natasha Merat, Don Norman, Thomas B. Sheridan, and Neville A. Stanton. (2021). Vulnerable road users and the coming wave of automated vehicles: Expert perspectives. Transportation Research Interdisciplinary Perspectives, 9, (Mar. 2021), 100293. DOI: https://doi.org/10.1016/j.trip.2020.100293

[54] Liu Tang and Jia Zhou. 2020. Usability assessment of augmented reality-based pedestrian navigation aid. In 22nd HCI International Conference (HCII 2020), July 19 - 24, 2020, Copenhagen, Denmark, 581-591. DOI: https://doi.org/10.1007/9783-030-49904-4_43

[55] Techcrunch. 2021. Microsoft gets contract worth up to $\$ 22$ billion to outfit US Army with 120,000 AR headsets. (March 2021). Retrieved April 1, 2021 from https://techcrunch.com/2021/03/31/microsoft-wins- contract-worth-up-to22-billion-to-outfit-u-s-army-with-120000-ar-headsets

[56] 3DScannerApp. 2021. 3D Scanner App - LIDAR Scanner for iPad \& iPhone Pro. (March 2021). Retrieved March 30, 2021 from https://www.3dscannerapp.com/

[57] Maureen Troel-Madec, Laurence Boissieux, Stan Borkoswki, Dominique Vaufreydaz, Julien Alaimo, Sandrine Chatagnon, and Anne Spalanzani. 2019. EHMI positioning for autonomous vehicle/pedestrians interaction. In Proceedings of the 31st Conference on l'Interaction Homme-Machine: Adjunct (IHM '19). Association for Computing Machinery, New York, NY, USA, Article 1, 1-8. DOI: https://doi.org/10.1145/3366551.3370340

[58] Pamela S. Tsang and Michael A. Vidulich. 2002. Principles and Practice of Aviation Psychology. CRC Press.

[59] Umbrellium. 2017. Umbrellium develops light-up crossing that only appears when needed. (October 2017). Retrieved March 25, 2021 from https://www.dezeen.com/2017/10/12/umbrellium-develops-interactiveroad-crossing-that-only-appears-when-needed-technology.

[60] Unity. 2020. MARS Direct Placement Example. (June 2020). Retrieved Apri 26, 2021 from https://forum.unity.com/threads/mars-direct-placement-example. 908381

[61] Unity. 2021. Unity MARS Overview. (March 2021). Retrieved April 26, 2021 from https://docs.unity3d.com/Packages/com.unity.mars@1.3/manual/index.htm

[62] Unity. 2021b. Unity MARS Companion app - Open Beta announcement. (Jan 2021). Retrieved March 30, 2021 from https://forum.unity.com/threads/unitymars-companion-app-open-beta-announcement.1037638/

[63] Ziran Wang, Kyungtae Han, and Prashant Tiwari. 2020. Augmented realitybased advanced driver-assistance system for connected vehicles. In 2020 IEEE International Conference on Systems, Man, and Cybernetics (SMC), October 11 14, 2020, Toronto, ON, Canada, 752-759. DOI: https://doi.org/10.1109/SMC42975. 2020.9283462

[64] John P. Wann, Simon Rushton, and Mark Mon-Williams. 1995. Natural problems for stereoscopic depth perception in virtual environments. Vision Research 35, 19 (Oct. 1995), 2731-2736. DOI: https://doi.org/10.1016/0042-6989(95)00018-U

[65] Christopher D. Wickens, Justin G. Hollands, Simon Banbury, and Raja Parasuraman. 2015. Engineering Psychology and Human Performance. Psychology Press.

[66] Joost de Winter, Pavlo Bazilinskyy, Dale Wesdorp, Valerie de Vlam, Belle Hopmans, Just Visscher, and Dimitra Dodou. How do pedestrians distribute their visual attention when walking through a parking garage? An eye-tracking study. Ergonomics, (Jan. 2021), 1-16. DOI: https://doi.org/10.1080/00140139.2020.1862310

[67] Tieme P. Witte. 2020. Design of an Augmented Reality System for the Live Visualization of Ship Identification Data. Master's thesis.Delft University of Technology, Delft, The Netherlands. 